\title{
Dwarf-dwarf interactions: a common origin for dwarf Wolf-Rayet galaxies
}

\author{
César Esteban and David I. Méndez \\ Instituto de Astrofísica de Canarias, \\ E-38200 La Laguna, Tenerife, Canary Islands, Spain
}

\begin{abstract}
We present some results of an observational programme devoted to obtain deep imagery and high-resolution spectroscopy for a sample of dwarf Wolf-Rayet galaxies. Our main result is that interaction with fainter optical companions could be the origin of the strong star formation episodes observed in, at least, an important fraction of the objects. In all the cases presented, the companions and the signatures of interaction or merging can only be detected when deep and detailed observations are taken. We predict that the fraction of dwarf WR galaxies (or even $\mathrm{H}$ II galaxies in general) with interacting signatures will increase when detailed observations for more objects are available.
\end{abstract}

\section{Introduction}

$\mathrm{H}$ II galaxies and specially those classified as Wolf-Rayet galaxies are dwarf systems undergoing violent star formation. Once the SF has been initiated in a gas-rich galaxy, the stochastic self-propagating processes could maintain the high SF rate and its spatial propagation inside the galaxy (see Gerola et al. 1980). However, dwarf galaxies cannot sustain a spiral density wave nor disk instabilities and, therefore, the initial SF process seems to need an external mechanism to be triggered.

Several authors have proposed that interactions might be important in the triggering of SF and the evolution of $\mathrm{H}$ II galaxies. Campos-Aguilar \& Moles (1991), Campos-Aguilar et al. (1993) and Telles \& Terlevich (1995) checked for the presence of massive optical companions around large samples of dwarf galaxies finding that the indications for interactions with larger objects are very scarce. It is surprising that Telles \& Terlevich (1995) found that galaxies with most disturbed optical morphology have no bright neighbors.

On the other hand, Taylor et al. $(1993,1995,1996)$ have carried out a search for gas-rich $\mathrm{HI}$ companions to a sample of $\mathrm{H}$ II galaxies. They found that out of 20 objects, 12 have at least one companion with similar or lower H I masses. Moreover, in a recent radio survey of five barred Magellanic spiral galaxies Wilcots et al. (1996) have found that 4 out of 5 present a dwarf H I companion. These authors suggest that the interaction with those fainter companions could be the mechanism for the formation of the bars. Finally, Brinks (1997) concludes that $\mathrm{H}$ II galaxies do have gas-rich $\mathrm{H}$ I companions of similar or lower masses with an observed probability at least of the order of $60 \%$.

The presence of WR stars in a dwarf galaxy is a clear indication that the SF is very recent or is still ongoing in the object. If we are interested in the investigation of the mechanisms that trigger SF in these objects, dwarf WR galaxies are 
the ideal systems. The catalogue of WR galaxies compiled by Conti (1991) contains objects of different nature as spiral galaxies, $\mathrm{H}$ II regions in galaxies, blue compact dwarves, and others. Among these, there are some objects classified as merging or interacting galaxies. Most of them are interacting spiral galaxies as NGC 1614, NGC 1741 (H 31A and C), ESO 148-IG02, and NGC 7714. But only a few of them are truly dwarves as NGC 1510, which is an amorphous dwarf galaxy interacting with a nearby larger barred spiral (Eichendorf \& Nieto 1984); and II Zw 40, a typical blue compact dwarf galaxy which is probably the product of the collision of two H I clouds of similar mass (Brinks \& Klein 1988).

Although interaction between low-mass $\mathrm{H}$ I objects seems to be a promising scenario for explaining the triggering of SF in $\mathrm{H}$ II galaxies, the possibility of interactions between two (or more) optical low-mass galaxies have not yet been explored in depth and the examples are very few and not definitive. In this paper, we present and discuss some published and new data for dwarf WR galaxies that change dramatically the situation. These results indicate that interaction and merging with optical low-mass companions is a rather general situation that could explain, very probably, the recent violent SF activity we are witnessing in many or most of these galaxies.

\section{Evidence for interactions with optical low-mass companions}

The first example we are going to discuss is $\mathrm{IZw} 18$, which is known to be the most metal deficient BCDG. It has been added very recently to the list of WR galaxies by Izotov et al. (1997) and Legrand et al. (1997), due to the discovery of few WR stars from the spectrum of the NW region of this galaxy. Dufour et al. $(1996 \mathrm{a}, 1996 \mathrm{~b})$ from deep HST images found that the near diffuse feature (feature $\mathrm{C}$ ) to the $\mathrm{NW}$ of $\mathrm{IZw} 18$ appeared to be a faint irregular galaxy, possibly a companion stellar system. Deep intermediate-resolution spectra passing through the brightest part of feature $\mathrm{C}$ obtained by Dufour et al. (1996c) have revealed that feature $\mathrm{C}$ is at the same radial velocity as the main body of $\mathrm{I} Z \mathrm{~W} 18$, indicating that they are truly companion systems and a physical connection of the ionized gas between them. Dufour et al. (1996c) found a smoothly varying double-sinusoidal velocity field in the ionized gas along the axis crossing both objects. This complex behavior could be produced by dynamical interaction between both objects, however, other explanations cannot be rejected.

Another example of possible interaction between an $\mathrm{H}$ II galaxy and a very faint optical companion is Mrk 1094. Brinks (1990) discovered a H I companion located $51 \mathrm{kpc}$ to the $\mathrm{S}$ of the main galaxy. Walter et al. (1997) found that the companion is very faint in the optical, with a B luminosity an order of magnitude lower than Mrk 1094. The optical morphology of Mrk 1094 resembles that of a barred galaxy with two short apparent spiral arms at its ends. This makes that Mrk 1094 is probably at the upper end of which is usually considered a dwarf galaxy. Walter et al. (1997) carried out a dynamical study of the object estimating that the time elapsed since the perigalacticon of the $\mathrm{H}$ I companion is about $3 \times 10^{8}$ years. It is interesting to point out that the $\mathrm{N}$-body simulations for tidal interactions of disk galaxies by Noguchi \& Ishibashi (1986) predict that bars are produced by interactions, and that the maximum activity of the SF takes place about $3 \times 10^{8}$ years after the perigalacticon, in excellent agreement 


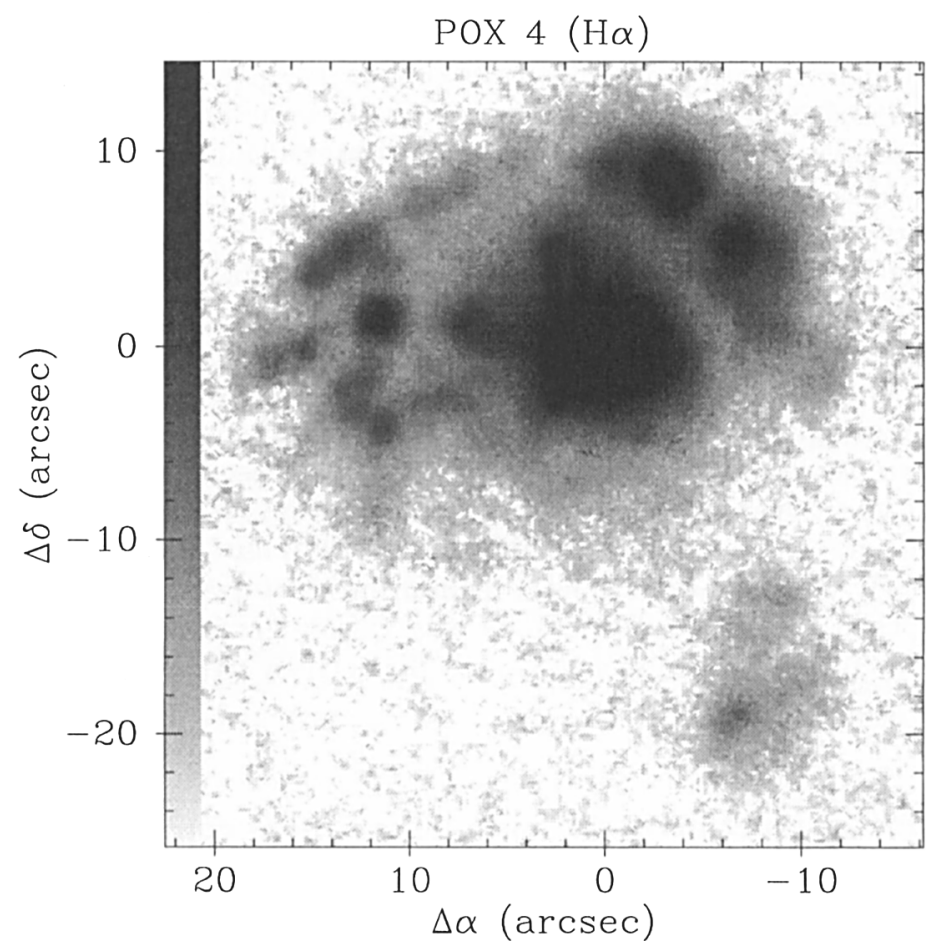

Figure 1. Continuum-subtracted $\mathrm{H} \alpha$ image of POX 4. $\mathrm{N}$ is at the top and $\mathrm{E}$ on to the left. Image taken from Méndez \& Esteban (1999).

with the results by Walter et al. (1997). A detailed study of the recent SF history in this galaxy can be found in Méndez et al. (1999a).

IZw 18 and Mrk 1094 are two clear examples for the presence of fainter optical companions around $\mathrm{H}$ II galaxies, however, the evidences of interaction between them are indirect or still ambiguous. In a long-term project devoted to obtain deep imagery and spectroscopy of a sample of dwarf WR galaxies, we have found that an important fraction of the objects show direct or even dramatic observational evidences for the real existence of such interactions with fainter optical companions. In the following, we will discuss the most dramatic examples: POX 4, Tol 35 , and Zw 0855+06.

POX 4 is a typical BCDG. Its WR nature was discovered by Campbell et al. (1986). We have obtained deep $\mathrm{H} \alpha$ and $U B V$ CCD images as well as highresolution spectroscopy of the galaxy (all the data presented in this paper were collected with the $2.5 \mathrm{~m}$ Nordic Optical Telescope, NOT, at the Observatorio del Roque de Los Muchachos in La Palma). The results and their discussion will be published in Méndez \& Esteban (1999). In Figure 1 we show the $\mathrm{H} \alpha$ image of POX 4. The morphology of the galaxy consists of a group of several starforming knots arranged in a rough ring-like structure, with the brightest knot slightly off-center. The morphology in the broad-band filters is very similar to that in the $\mathrm{H} \alpha$ emission. It is remarkable the presence of an irregular low-surface brightness companion located about $20^{\prime \prime} 5$ (4.7 kpc at the distance of $47.3 \mathrm{Mpc}$ ) 
to the SW along the minor axis of the galaxy. This companion is also visible in the broad-band images and has a fainter and diffuse extension to the $\mathrm{N}$, in the direction towards POX 4.

All the observational results gathered for POX 4 lead us to interpret this object as a member of the uncommon class of ring galaxies, in particular a lowmass analog of the well-known Cartwheel galaxy. Following Lynds \& Toomre (1976) and Toomre (1978), ring galaxies are the product of head-on collisions of a compact intruder object onto a disk galaxy. In the case of POX 4, we have the following observational results that match the proposed scenario:

- The irregular companion galaxy (the intruder) is located along the minor axis of the galaxy and shows a redshifted velocity of $130 \mathrm{~km} \mathrm{~s}^{-1}$ with respect to the main object. Assuming a circular disk for the shape of the main galaxy, an inclination angle of about $48^{\circ}$ is derived. With this angle, and taking into account a linear radius of $3.4 \mathrm{kpc}$, we estimate that the encounter should have occured about $3.2 \times 10^{7}$ years ago.

- The companion galaxy is an extremely faint object but emits in $\mathrm{H} \alpha$ and has very blue colors, both facts indicate that the companion has suffered (or is suffereing) a recent SF process, very probably due to the collision.

- The position slightly off-center of the dominant knot is expected in the most general case of an impact parameter different from zero in the passage of the intruder.

- The high resolution spectra of POX 4 obtained by Méndez \& Esteban (1997; see their Figure 8) show that the galaxy is rotating along its major axis and indicate that could be expanding. Taking into account the geometry of the object we estimate a rotation velocity of $61-91 \mathrm{~km} \mathrm{~s}^{-1}$ and an expansion velocity of $79-14 \mathrm{~km} \mathrm{~s}^{-1}$ depending on whether the dominant knot is at the center or at the edge of the ring respectively. The range of expansion velocities obtained from the spectra is consistent with the expected upper limit of the expansion velocity of $104 \mathrm{~km} \mathrm{~s}^{-1}$ estimated from simply dividing the radius of the galaxy by the estimated $3.2 \times 10^{7}$ years elapsed since the collision.

Our second object, Tol 35, has been traditionally classified as a BCDG. Kunth \& Joubert (1985) found an emission feature et $4686 \AA$ attributable to the presence of WR stars in the galaxy. As in the case of POX 4, we have obtained deep $\mathrm{H} \alpha$ and broad-band $(U B V)$ CCD images of the object as well as high-resolution echelle spectra. The results will be also published in Méndez \& Esteban (1999). In Figure 2 we show the $\mathrm{H} \alpha$ image of Tol 35. The high-intensity levels image (left) shows the presence of several knots of SF and a closed loop to the SW and centered on the central brightest knot. This curious feature is only seen in the $\mathrm{H} \alpha$ image and, therefore, it should be of gaseous nature. At low intensities (right) there is indication of the presence of the beginning of two possible crossed tidal tails coincident with the loop. This fact suggests that the loop could be simply the bright intersection of both tails resembling, in a smaller scale, the inner part of the tails of the Antennae (see Whitmore \& Schweizer 1995). 

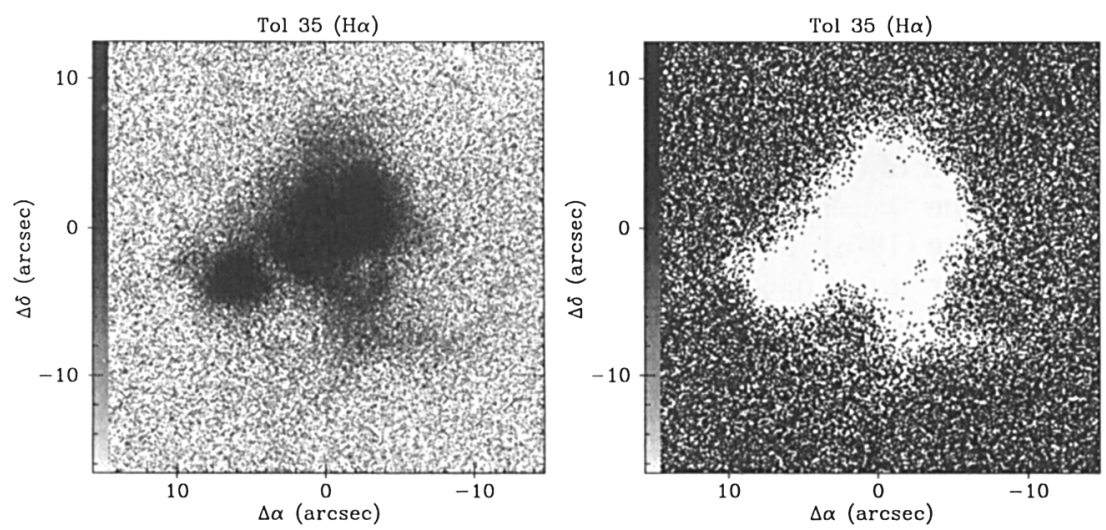

Figure 2. Continuum-subtracted $\mathrm{H} \alpha$ image of Tol 35. Left: high-intensity levels. Right: low-intensity levels. $\mathrm{N}$ is at the top and $\mathrm{E}$ on the left. Images taken from Méndez \& Esteban (1999).

Taking into account all the observational results for this object, we consider that there are, at least, three pieces of evidence to interpret Tol 35 as a recent merger of two gaseous-rich dwarf galaxies:

- The presence of a strong burst of SF in the object and a large number of WR stars is an indication of the extreme youth of the burst or that the $\mathrm{SF}$ is still ongoing in the galaxy.

- The presence of crossed tidal tails is a strong argument for the interacting nature of Tol 35. Since the pioneering works by Toomre (1970) and Toomre \& Toomre (1970), it is well known that gravitational interactions between galaxies could generate tidal features. In particular, the mutual inclination of two rotating and interacting disk can create apparent tail crossings. The fact that they are only visible $\mathrm{H} \alpha$ imply that they are of gaseous nature. Therefore, the interaction should be between to gas-rich disks.

- The high-resolution spectrum taken along the major axis of the galaxy shows the presence of different motion systems coexisting in the galaxy. In fact, the brightest central knot is redshifted about $50 \mathrm{~km} \mathrm{~s}^{-1}$ with respect to the rest of the knots. This is an evidence that the merging is in an intermediate stage of evolution (see Hibbard \& van Gorkom 1996) and has not reached yet a relaxed dynamical situation.

Finally, our third object shows the most dramatic and clear evidence that major interactions are possible between two dwarf galaxies. Zw $0855+06$ is an emission line dwarf galaxy discovered by Zwicky and included as an un-named object in his catalogue (Zwicky 1971). Vacca \& Conti (1992) detected the presence of about $110 \mathrm{WR}$ stars in its spectrum. We have obtained $\mathrm{H} \alpha$, and $B V$ CCD images as well as a high-resolution spectrum of $\mathrm{Zw} 0855+06$. All the results and their discussion are presented in Méndez et al. (1999b). In Figure 3 we show a deep $\mathrm{V}$ image of the object. The most remarkable feature of the image 


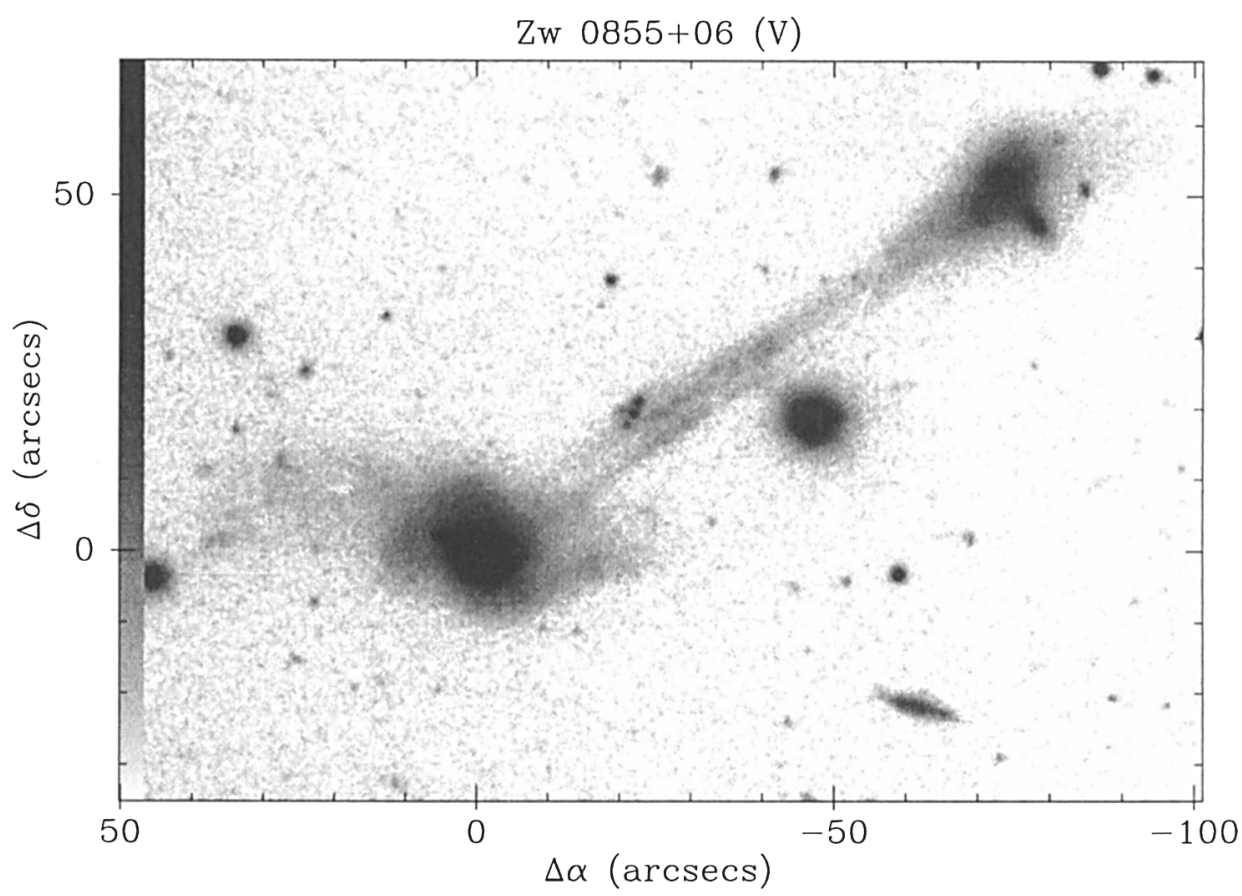

Figure 3. Deep $V$ image of $\mathrm{Zw} 0855+06$ and its companion. The intensity scale is logarithmic. $\mathrm{N}$ is at the top and $\mathrm{E}$ on the left. Image taken from Méndez et al. (1999b).

is the presence of a very straight bridge $\left(\sim 65^{\prime \prime}=14.7 \mathrm{kpc}\right.$ at the distance of $46.8 \mathrm{Mpc}$ ) connecting $\mathrm{Zw} 0855+06$ and a fainter companion located at the NW. It is also interesting the existence of two diffuse plumes towards the $\mathrm{E}$ and $\mathrm{W}$ of the main galaxy. The companion galaxy, that we shall refer to as $\mathrm{Zw} 0855+06 \mathrm{~B}$, has another smaller companion object very close to the $\mathrm{SW}(\mathrm{Zw} 0855+06 \mathrm{C})$. The main galaxy has very blue color $(B-V=0.19)$ which gives an age consistent with the $\mathrm{W}(\mathrm{H} \alpha)$ and about $5-6 \times 10^{6}$ years applying the models by Leitherer $\&$ Heckman (1995). The $B-V$ color of $\mathrm{Zw} 0855+06 \mathrm{~B}$ is about 0.49 . Applying the models by Bruzual and Charlot (1996), we find that this color is consistent with an instantaneous burst about $10^{9}$ years old. On the other hand, the third galaxy, $\mathrm{Zw} 0855+06 \mathrm{C}$, has a much more redder color, indicating that it is probably a highly reddened edge-on dwarf elliptical galaxy. Profile fittings of the main galaxy and $\mathrm{Zw} 0855+06 \mathrm{~B}$ indicate that both objects have exponential disks. Neither $\mathrm{Zw} 0855+06 \mathrm{~B}$ nor the bridge are detected in the $\mathrm{H} \alpha$ image.

The bridge has a $B-V$ color similar to $\mathrm{Zw} 0855+06 \mathrm{~B}$. This fact, its straight shape and alignment with the companion galaxy, indicate that the bridge (which is composed mainly of stars) has been formed by the disruption of the disk of Zw $0855+06 \mathrm{~B}$ due to the interaction with the WR galaxy. On the other hand, the presence of diffuse plumes in the main galaxy indicates that it is seen in a probably very oblique plane with respect to the plane of the encounter. It is remarkable the presence of several small knots along the bridge with $B-V$ 
color similar to the main galaxy and bluer than the rest of the bridge. This fact suggests the presence of an episode of in situ SF in the bridge after its creation. SF activity in other tidal tails has been detected in other interacting galaxies (see Schombert et al. 1990).

\section{Epilogue}

As can be seen in these examples, the interaction between dwarf galaxies is, at least, a common phenomenon. POX 4 and Tol 35, considered as isolated objects in previous works, have been identified as interacting objects after detailed deep, high-resolution images and spectra have been obtained and analysed. Previous works, devoted to obtain statistical properties of large samples of dwarf galaxies, did not achieve enough detail to discover the ultimate interacting nature of the objects.

Finally, it is very significant that of 14 dwarf WR galaxies (taken from the catalogue by Conti 1991) for which we have observations, about 8 of them (more than $50 \%$ ) present clear or possible evidences of interaction or merging with other fainter objects. We are collecting additional data for part of the objects and specially high-resolution spectra for most of them. Therefore, the final fraction of objects with interacting nature could be even larger. In the meantime, we can speculate: are WR features in dwarf galaxies signatures of interaction?.

Acknowledgments. We are grateful to M. Balcells and P.S. Conti for fruitful "interactions". CE thanks the financial support of project PB97-0218 of the Dirección General de Investigación Científica y Técnica of the Spanish Ministerio de Educación y Ciencia.

\section{References}

Brinks, E. 1990, in: R. Wielen (ed.), Dynamics and Interactions of Galaxies (Berlin: Springer), p. 146

Brinks, E. 1997, RevMexAA-SC 6, 14

Brinks, E., Klein, U. 1988, MNRAS 231, 63P

Bruzual, G., Charlot, S. 1996, in: Leitherer et al. 1996, PASP 108, 996

Campbell, A.W., Terlevich, R., Melnick, J. 1986, MNRAS 223, 811

Campos-Aguilar, A., Moles, M. 1991, A\&A 241, 358

Campos-Aguilar, A., Moles, M., Masegosa, J. 1993, AJ 106, 1784

Conti, P.S. 1991, ApJ 377, 115

Dufour, R.J., Garnett, D.R., Skillman, E.D., Shields, G.A. 1996a, in: C. Leitherer, U. Fritze-von Alvenslaben \& J. Huchra (eds.), From Stars to Galaxies: The Impact of Stellar Physics on Galaxy Evolution ASP-CS 98, 358

Dufour, R.J., Garnett, D.R., Skillman, E.D., Shields, G.A. 1996b, in: P. Benvenuti, F.D. Machetto \& E.J. Schreier (eds.), Science with the Hubble Space Telescope. II (Baltimore: STScI), p. 348

Dufour, R.J., Esteban, C., Castañeda, H.O. 1996c, ApJ 471, L87

Eichendorf, W., Nieto, J.-L. 1984, A\&A 132, 342

Gerola, H., Seiden, P.E., Schulman, L.S. 1980, ApJ 242, 544 
Hibbard, J.E., van Gorkon, J.H. 1996, AJ 111, 655

Izotov, Y.I., Folt, C.B., Green, R.F., Guseva, N.G., Thuan, T.X. 1997, ApJ 487, L37

Kunth, D., Joubert, M. 1985, A\&A 142, 411

Legrand, F., Kunth, D., Roy, J.-R., Mas-Hesse, J.M., Walsh, J.R. 1997, A\&A 326, L17

Leitherer, C., Heckman, T.M. 1995, ApJS 96, 9

Lynds, R., Toomre, A. 1976, ApJ 209, 382

Méndez, D.I., Esteban, C. 1997, ApJ 488, 652

Méndez, D.I., Esteban, C. 1999, A\&A submitted

Méndez, D.I., Cairós, L.M., Esteban, C., Vílchez, J.M. 1999a, AJ 117, 1688

Méndez, D. I., Esteban, C., Balcells, M. 1999b, AJ 117, 1229

Noguchi, M., Ishibashi, S. 1986, MNRAS 219, 670

Schombert, J.M., Wallin, J.F., Struck-Marcell, C. 1990, AJ 99, 497

Taylor, C., Brinks, E., Skillman, E.D. 1993, AJ 105, 128

Taylor, C., Brinks, E., Grashuis, R.M., Skillman, E.D. 1995, ApJS 99, 427

Taylor, C., Thomas, D., Brinks, E., Skillman, E.D. 1996, ApJS 107, 143

Telles, E., Terlevich, R. 1995, MNRAS 275, 1

Toomre, A. 1970, in: W. Becker \& G. Contopoulos (eds.), The Spiral Structure of Our Galaxy, Proc. IAU Symp No. 38, (Dordrecht: Reidel), p. 334

Toomre, A. 1978, in: M. Longair \& J. Einasto (eds.), The Large Scale Structure of the Universe, Proc. IAU Symp No. 79 (Dordrecht: Reidel), p. 109

Toomre, A., Toomre, J. 1972, ApJ 178, 623

Vacca, W.D., Conti, P.S. 1992, ApJ 401, 543

Walter, F., Brinks, E., Duric, N., Klein, U. 1997, AJ 113, 2031

Whitmore, B.C., Schweizer, F. 1995, AJ 109, 960

Wilcots, E.M., Lehman, C., Miller, B. 1996, AJ 111, 1575

Zwicky, F. 1971, Catalogue of Selected Compact Galaxies and Post-Eruptive Galaxies (Guemligen: F. Zwicky)

\section{Discussion}

Schulte-Ladbeck: Could you give us your definition of a companion? We (SchulteLadbeck \& Hopp 1998, AJ 116, 2886) often find star-forming regions that have the same appearance as your companions, but in very deep images turn out to be within the Holmberg diameter, so they are simply outlying regions of that same galaxy.

Esteban: Of course, the definition of companion objects is a problem. The point is that when we are witnessing a recent interaction or merging, the companion or intruder could be very near the main galaxy. The presence of interaction features or different motion systems in the objects can be interpreted as the effect of an interaction between the objects independently of their relative distance.

Joseph: How was your sample selected? Those of us who have been interested in the effects of interactions generally have not been very careful about using well-controlled samples.

Esteban: The sample was not specifically designed to search for interactions. In fact, the original reason was to analyse the ionized-gas morphology and dynamics of those WR galaxies, which have been classified as $\mathrm{H}$ II galaxies. The finding of clear interaction in some objects of the sample has been an unexpected result. 